\title{
GMR
}

\section{An experimental study on the use of icariin for improving thickness of thin endometrium}

\author{
A.W. Le ${ }^{1}$, Z.H. Wang ${ }^{1}$, X.Y. Dai ${ }^{1}$, T.H. Xiao ${ }^{1}$, R. Zhuo ${ }^{1}$, B.Z. Zhang ${ }^{2}$, \\ Z.L. Xiao ${ }^{2}$ and X.J. Fang ${ }^{2}$ \\ ${ }^{1}$ Department of Obstetrics and Gynecology, Affiliated Shenzhen Nanshan \\ People's Hospital of Guangdong Medical University, Shenzhen, China \\ ${ }^{2}$ Shenzhen Institutes of Advanced Technology, Chinese Academy of Sciences, \\ Shenzhen, China \\ Corresponding author: X.J. Fang \\ E-mail: leaiwen@126.com \\ Genet. Mol. Res. 16 (1): gmr16019126 \\ Received September 1, 2016 \\ Accepted November 16, 2016 \\ Published January 23, 2017 \\ DOI http://dx.doi.org/10.4238/gmr16019126
}

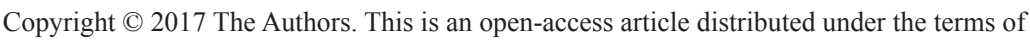
the Creative Commons Attribution ShareAlike (CC BY-SA) 4.0 License.

\begin{abstract}
This study aimed to investigate the effect of icariin (ICA) on thin endometrium in a rat model. To this end, 6- to 8-weekold female Sprague Dawley rats (105) were randomly divided into 7 groups: untreated, vehicle-treated (lavage with $\mathrm{NaCl}$ ), high-dose ICA (lavage with ICA at $200 \mathrm{mg} \cdot \mathrm{kg}^{-1} \cdot \mathrm{day}^{-1}$ ), medium-dose ICA (lavage ICA at $100 \mathrm{mg} \cdot \mathrm{kg}^{-1} \cdot \mathrm{day}^{-1}$ ), low-dose ICA (lavage with ICA at $50 \mathrm{mg} \cdot \mathrm{kg}^{-}$ ${ }^{1} \cdot$ day $^{-1}$ ), sham model (injected with $\mathrm{NaCl}$ at uterus horn), and sample group. To induce thin endometrium, rats of all groups (except shammodel) were injected with $95 \%$ ethanol via the uterine horn. Each group underwent its respective treatment for 3 estrous cycles, after which 5 rats from each group were sacrificed, and endometrial thickness was measured. The expression of CD31, factor VIII, vascular endothelial growth factor (VEGF), cytokeratin (CK), and vimentin were detected via immunohistochemistry. The results showed that CD31, factor VIII,
\end{abstract}

Genetics and Molecular Research 16 (1): gmr16019126 
and VEGF were primarily expressed in the cytoplasm of endometrial and vascular epithelial cells. No difference in the expression of these factors was detected between the ICA lavage groups and the untreated groups. However, high dose ICA-treated group exhibited significantly higher expression of CD31, factor VIII, and VEGF compared to that in the low dose and vehicle-treated groups. CK and vimentin in the endometrial tissue were significantly higher in the untreated and treatment groups compared to the vehicle-treated group. This study demonstrated that ICA increases thickness of the endometrium, and it may modulate expression of VEGF, CD31, and factor VIII.

Key words: Icariin; Thin endometrium; Factor VIII; CD31; Vascular endothelial growth factor (VEGF)

\section{INTRODUCTION}

Thin endometrium is a common gynecological problem; however, it still lacks a uniform definition. When endometrial thickness is $<7-8 \mathrm{~mm}$, there is a significant decrease in pregnancy rates (Al-Ghamdi et al., 2008; Shufaro et al., 2008; Aydin et al., 2013; Kasius et al., 2014). An endometrial thickness of $<7 \mathrm{~mm}$, measured using B-mode ultrasound during the mid-luteal phase (6-10 days following ovulation), is considered a thin endometrium.

Phytoestrogens (PE) are non-steroid compounds that exert a weak estrogenic effect when bound to estrogen receptors. Icariin (ICA), one of the active ingredients in PE, does not affect or exert anti-estrogenic effects on endometria of normal thickness (Xue et al., 2012; Gleicher et al., 2013; Lebovitz and Orvieto, 2014; Luo et al., 2015). However, its effects on thin endometria are unclear. Chung et al. (2008) found that ICA could promote angiogenesis through activation of the MEK/ERK and PI3K/Akt/eNOS signaling pathways. As clinical treatments of thin endometrium with ICA have been effective, we used a rat model of thin endometrium to investigate whether ICA promotes angiogenesis by increasing the expression of CD31, factor VIII, and vascular endothelial growth factor (VEGF).

\section{MATERIAL AND METHODS}

\section{Experimental animals}

In this study, 6- to 8-week-old specific pathogen free (SPF) female Sprague Dawley (SD) rats weighing $200 \pm 30 \mathrm{~g}$ were obtained from the Hunan SJA Laboratory Animal Co. Ltd., Changsha, China. Animals were housed in a SPF grade barrier system under a $12 \mathrm{~h} \mathrm{light/12}$ $\mathrm{h}$ dark cycle. The room temperature was maintained at $20^{\circ}-26^{\circ} \mathrm{C}$ with $50-60 \%$ humidity, and food and water were provided ad libitum. Experiments involving laboratory animals were conducted in strict accordance with the rules and guidelines of the Chinese Academy of Sciences Institutional Animal Care and Use Committee, Beijing, China.

\section{Experimental methods}

Thin endometrium in SD rats was established in the model group $(\mathrm{N}=15)$ by injecting

Genetics and Molecular Research 16 (1): gmr16019126 
$95 \%$ ethanol into the uterine horn, while the vehicle-treated group $(\mathrm{N}=15)$ was injected with saline. After establishment of the experimental model, vaginal smear tests were performed daily at 08:00 am, and 5 rats from each group were sacrificed after every 3rd estrous cycle. Uterine sections were stained with hematoxylin and eosin (H\&E), and endometrial thickness was assessed using the Image J software (National Institutes of Health, USA). Specifically, the vertical distance from the endometrial-myometrial junction to the uterine cavity was measured; the average of the measurements of the 4 thickest locations on each slide was defined as the endometrial thickness (Gao, 2011).

The remaining rats were simultaneously placed into cages at 4:00 pm in a 2:1 (male:female) ratio for mating. Vaginal plugs were examined daily to confirm successful mating, after which female and male rats were separated, and the female rats were reared individually. After 19 days, vaginal plugs were examined; pregnant rats were sacrificed followed by laparotomy to confirm intrauterine pregnancy.

ICA experiments were carried out on $105 \mathrm{SD}$ rats, which were randomly divided into 7 groups, each consisting of 15 rats. One group was randomly selected for injection with saline solution in the uterine horn (sham-model group). To induce thin endometrium, the rest of the rats (90) were injected with 95\% ethanol in their uterine horn. Rats in the sample group (15) were selected to confirm the establishment of the experimental model. The remaining rats were then divided into 5 additional groups: 3 ICA lavage treatment groups - high-dose $\left(200 \mathrm{mg} \cdot \mathrm{kg}^{-1} \cdot \mathrm{day}^{-1}\right)$, medium-dose $\left(100 \mathrm{mg} \cdot \mathrm{kg}^{-1} \cdot \mathrm{day}^{-1}\right)$, and low-dose $\left(50 \mathrm{mg} \cdot \mathrm{kg}^{-1} \cdot \mathrm{day}^{-1}\right)$ groups; a vehicle-treated group that was administered normal saline; and an untreated group. After the 3rd estrous cycle, 5 rats from each group were sacrificed to measure the endometrial thickness and to examine the histopathology.

We then implemented several improvements to the study design: 1) intravenous push of chloral hydrate was performed at a slower rate, and attempts were made to succeed in the first puncture; 2) depilatory cream was used to remove abdominal hair of rats to prevent abdominal cavity adhesions by residual pelage; 3 ) following injections, a syringe was used to aspirate the ethanol from the uterine horn, which was also repeatedly rinsed with saline; 4) the top surface was kept dry during surgery, and several layers of sterile gauze with saline were used on the lower layers to protect the surgical incision and prevent leakage of ethanol into the abdominal cavity; 5) following surgery, rats were kept in individual cages, and beddings were changed. Food was restrained (only provided after the first bowel movement), but rats were given free access to water. We noted that ICA worked well as a suspension and was not affected by fasting. All rats survived, and no difference in weight or estrous cycle was observed among different groups owing to the use of the above improvements.

\section{Specimen collection and treatment}

Animals were anesthetized via intraperitoneal injection of $10 \%$ chloral hydrate ( 350 $\mathrm{mg} / \mathrm{kg}$ ), and uterine tissue was excised via laparotomy. Blood-stained and adipose tissues were removed. After residual blood was washed with saline, tissues were fixed in Bouin's fixative (saturated aqueous picric acid solution:formaldehyde:glacial acetic acid, 15:5:1), and placed on a shaker for $24 \mathrm{~h}$. Tissue sections were embedded in paraffin, stained with H\&E, and were analyzed by immunohistochemistry.

\section{Paraffin embedding, tissue sectioning, H\&E staining, and immunohistochemistry}

For histological analysis, tissues were embedded in paraffin and sectioned

Genetics and Molecular Research 16 (1): gmr16019126 
with a microtome at $5-\mu \mathrm{m}$ thickness. The sections were flattened in the water bath and mounted onto slides, which were dried overnight at $37^{\circ} \mathrm{C}$ in an oven. Tissue sections were deparaffinized and rehydrated, stained by H\&E. They were then dehydrated again, and mounted on glass slides for imaging.

For immunohistochemistry studies, following deparaffinization and rehydration, sections were blocked with $1 \%$ BSA PBS for $1 \mathrm{~h}$ at room temperature. They were then incubated with primary antibodies for VEGF, CD31 and factor VIII separately at $4^{\circ} \mathrm{C}$ overnight. This was followed by staining with HRP-conjugated secondary antibody for $1 \mathrm{~h}$ at room temperature. VEGF, CD21, and factor VIII were visualized by DAB staining. Images were captured on the light microscope with a CCD camera. The average optical density (AOD) of each protein was analyzed using the ImagePro Plus 7.0 software (MediaCybernetics, USA).

\section{Statistical analysis}

Measurements are reported as means \pm standard deviation. The Student $t$-tests were used to compare differences among 2 groups, and one-way ANOVA was used for comparisons between 3 or more groups. Count data were analyzed using the chi-square test. Using the SPSS 17.0 software (SPSS version 17.0; SPSS, Inc., Chicago, IL), P values were analyzed for two-tailed probability, with $\mathrm{P}<0.05$ representing statistical significance.

\section{RESULTS}

\section{H\&E staining of rat endometrial tissues}

After appropriate treatments were administered for 3 estrous cycles, H\&E staining was performed on rat samples from each group (Figure 1). We found that the endometrial epithelium consisted mostly of monolayered columnar cells in regular arrangements. Following ethanol treatment, endometrial epithelial cells became stratified and exhibited vigorous growth; glands were tubular, while glandular epithelial cells were columnar and cubic. Glandular secretion was strong with mild interstitial edema (groups A, B, and C). In the vehicle-treated group (group E), all layers of the uterine wall had become thinner, especially in the endometrial layer. Abnormalities were observed in parts of the endometrial epithelium, which took on the appearance of a simple squamous epithelium. In addition, the nuclear-cytoplasmic ratio was found to be abnormal. The glandular epithelium of the treated rats was similar as compared to that of normal rats, which showed an evenly distributed and intact monolayer of columnar cells with regular arrangements. Quantification of endometrium thickness in each group is shown in Table 1.

\section{Expression of CD31, factor VIII, and VEGF in endometrial tissue}

Positive staining (light or dark brown granules) were observed in the cytoplasm and the nucleus. CD31 was mainly expressed in the cytoplasm of vascular endothelial cells and endometrial epithelial cells (Figure 2). Factor VIII (Figure 3) and VEGF (Figure 4) were expressed in the cytoplasm of endometrial, glandular, and vascular endothelial cells. As shown in Tables 2-4, there was no significant difference in the AOD of CD31, factor VIII, and VEGF between the medium/ high dose groups and the untreated group.

Genetics and Molecular Research 16 (1): gmr16019126 

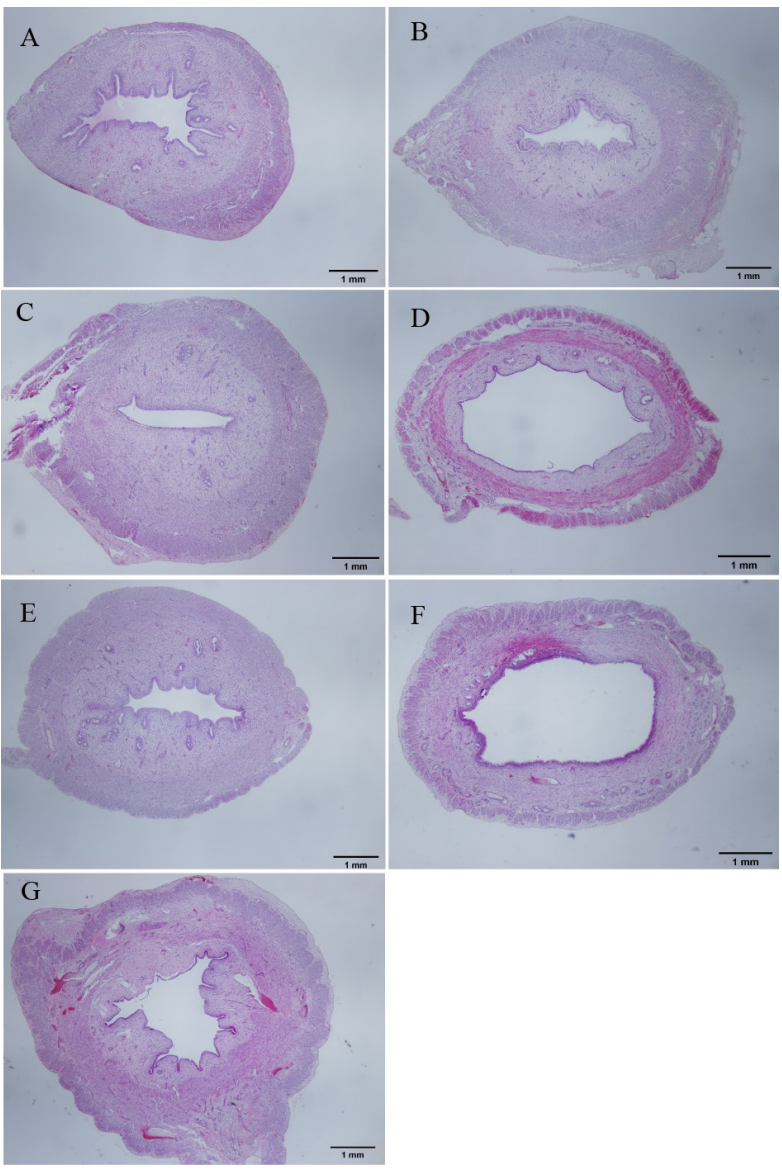

Figure 1. H\&E staining. Images are taken at $40 \mathrm{X}$ magnification. A-G. Low-dose ICA, medium-dose ICA, highdose ICA, control, blank, sample, sham, respectively.

Table 1. Comparison of endometrial thickness in each group.

\begin{tabular}{l|c|c}
\hline Group & Number & Mean endometrial thickness $(\mu \mathrm{m})$ \\
\hline Low-dose & 10 & $358.092 \pm 124.1734$ \\
\hline Medium-dose & 10 & $521.704 \pm 184.3222^{* * *}$ \\
\hline High-dose & 10 & $506.173 \pm 137.0697^{* * *}$ \\
\hline Vehicle-treated & 10 & $264.976 \pm 103.8369$ \\
\hline Untreated & 10 & $536.126 \pm 146.1215^{* * *}$ \\
\hline Sample & 10 & $328.343 \pm 146.1786$ \\
\hline Sham & 10 & $565.090 \pm 169.9738^{\Delta \Delta}$ \\
\hline
\end{tabular}

Data are reported as means $\pm \mathrm{SD} ; * * *$ statistically significant difference in endometrial thickness, as compared to vehicle-treated group $(\mathrm{P}<0.001)$; ${ }^{\Delta \Delta}$ statistically significant difference $(\mathrm{P}<0.01)$ in endometrial thickness, as compared to sample group.

However, the medium, high dose, and untreated groups exhibited significantly higher AOD in all factors as compared to the vehicle-treated group. No difference in AOD was observed between the low dose and the vehicle-treated groups.

Genetics and Molecular Research 16 (1): gmr16019126 


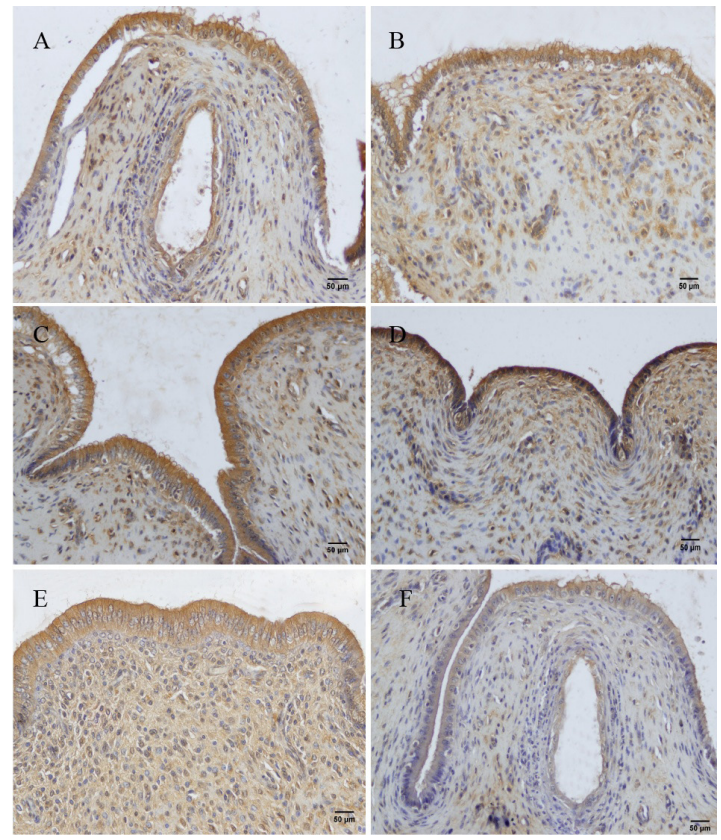

Figure 2. Protein expression of CD31 in the endometrium. Images are taken at $400 \mathrm{X}$ magnification. A-F. Low-dose ICA, medium-dose ICA, high-dose ICA, control, blank, CD31 antibody-negative.

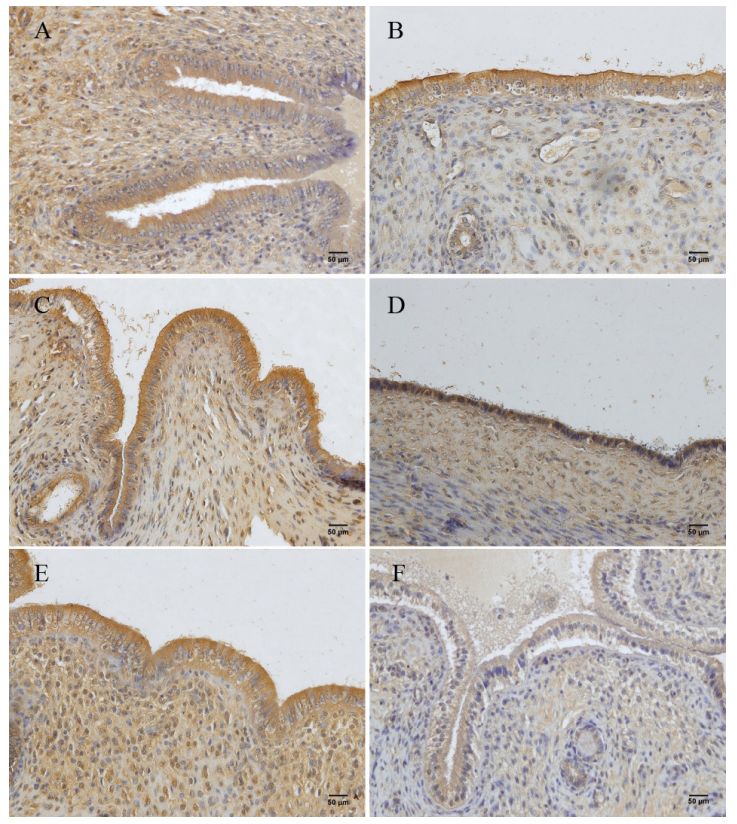

Figure 3. Protein expression of factor VIII in the endometrium. Images are taken at 400X magnification. A-F. Lowdose ICA, medium-dose ICA, high-dose ICA, control, blank, VIII antibody-negative.

Genetics and Molecular Research 16 (1): gmr16019126 


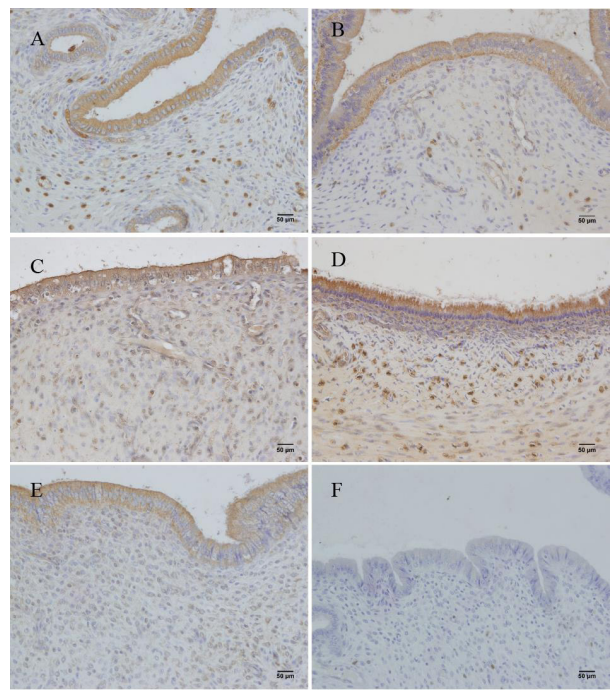

Figure 4. Protein expression of VEGF in the endometrium. Images are taken at 400X magnification. A-F. Lowdose ICA, medium-dose ICA, high-dose ICA, control, blank, VEGF antibody-negative.

Table 2. Average optical density of CD31 protein.

\begin{tabular}{l|c|c}
\hline Group & Number $(\mathrm{N})$ & Average optical density \\
\hline Low-dose & 10 & $0.47 \pm 0.89$ \\
\hline Medium-dose & 10 & $0.52 \pm 0.06^{* *}$ \\
\hline High-dose & 10 & $0.53 \pm 0.09^{* *}$ \\
\hline Vehicle-treated & 10 & $0.42 \pm 0.07$ \\
\hline Untreated & 10 & $0.52 \pm 0.07^{*}$ \\
\hline
\end{tabular}

Data are reported as means $\pm \mathrm{SD} ; * \mathrm{P}<0.05$ as compared to vehicle-treated group; $* * \mathrm{P}<0.01$ as compared to vehicle-treated group.

Table 3. Average optical density of factor VIII protein.

\begin{tabular}{l|c|c}
\hline Group & Number $(\mathrm{N})$ & Average optical density \\
\hline Low dose & 10 & $0.46 \pm 0.07$ \\
\hline Medium dose & 10 & $0.54 \pm 0.05^{* *}$ \\
\hline High dose & 10 & $0.57 \pm 0.10^{* * *}$ \\
\hline Vehicle-treated & 10 & $0.42 \pm 0.07$ \\
\hline Untreated & 10 & $0.56 \pm 0.08^{* *}$ \\
\hline
\end{tabular}

Data are reported as means $\pm \mathrm{SD} ; * * \mathrm{P}<0.01$ as compared to vehicle-treated group; $* * * \mathrm{P}<0.001$ as compared to vehicle-treated group.

Table 4. Average optical density of factor VEGF protein.

\begin{tabular}{l|c|c}
\hline Group & Number $(\mathrm{N})$ & Average optical density \\
\hline Low dose & 10 & $0.44 \pm 0.07$ \\
\hline Medium dose & 10 & $0.53 \pm 0.08^{* *}$ \\
\hline High dose & 10 & $0.54 \pm 0.09^{* *}$ \\
\hline Vehicle-treated & 10 & $0.42 \pm 0.09$ \\
\hline Untreated & 10 & $0.51 \pm 0.10^{*}$ \\
\hline
\end{tabular}

Data are reported as means $\pm \mathrm{SD} ; * \mathrm{P}<0.05$ as compared to vehicle-treated group; $* * \mathrm{P}<0.01$ as compared to vehicle-treated group.

Genetics and Molecular Research 16 (1): gmr16019126 


\section{Expression of CK and vimentin}

CK was mainly expressed in the cytoplasm of endometrial glandular epithelium and luminal epithelium (Figure 5). Vimentin was mainly expressed in the cytoplasm of mesenchymal stromal cells (Figure 6). There was no significant difference in the AOD of $\mathrm{CK}$ and vimentin among the low-, medium-, and high-dose groups and the untreated group. However, AOD was higher in the ICA and untreated groups as compared to the vehicle-treated group (Tables 5 and 6).

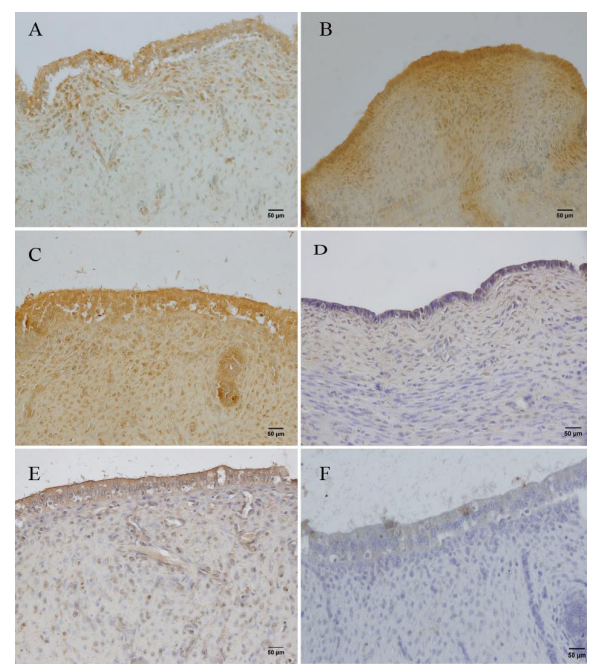

Figure 5. Protein expression of $\mathrm{CK}$ in the endometrial. Images are taken at 400X magnification. A-F. Low-dose ICA, medium-dose ICA, high-dose ICA, blank, CK antibody-negative.

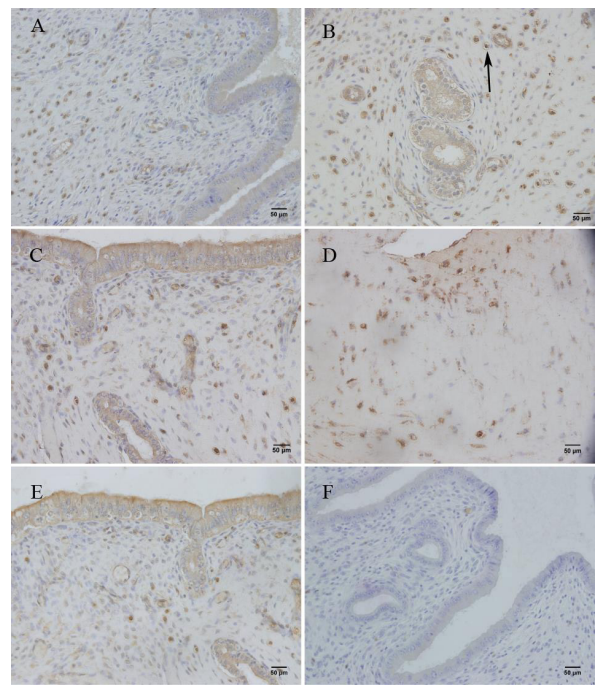

Figure 6. Protein expression of vimentin in the endometrium. Images are taken at 400X magnification. A-F. Lowdose ICA, medium-dose ICA, high-dose ICA, control, blank, vimentin antibody-negative.

Genetics and Molecular Research 16 (1): gmr16019126 


\section{Table 5. Average optical density of CK.}

\begin{tabular}{l|c|c}
\hline Group & Number $(\mathrm{N})$ & Average optical density \\
\hline Low dose & 10 & $0.49 \pm 0.08$ \\
\hline Medium dose & 10 & $0.55 \pm 0.13^{* *}$ \\
\hline High dose & 10 & $0.57 \pm 0.09^{* * *}$ \\
\hline Vehicle-treated & 10 & $0.40 \pm 0.06$ \\
\hline Untreated & 10 & $0.53 \pm 0.12^{*}$ \\
\hline
\end{tabular}

Data are reported as means $\pm \mathrm{SD} ; * \mathrm{P}<0.05$ as compared to vehicle-treated group; $* * \mathrm{P}<0.01$ as compared to vehicle-treated group; $* * * \mathrm{P}<0.001$ as compared to vehicle-treated group.

Table 6. Average optical density of vimentin.

\begin{tabular}{l|c|c}
\hline Group & Number $(\mathrm{N})$ & Average optical density \\
\hline Low dose & 10 & $0.47 \pm 0.08^{*}$ \\
\hline Medium dose & 10 & $0.49 \pm 0.08^{* *}$ \\
\hline High dose & 10 & $0.50 \pm 0.05^{* *}$ \\
\hline Vehicle-treated & 10 & $0.40 \pm 0.05$ \\
\hline Untreated & 10 & $0.47 \pm 0.07^{*}$ \\
\hline
\end{tabular}

Data are reported as means $\pm \mathrm{SD} ; * \mathrm{P}<0.05$ as compared to vehicle-treated group; $* * \mathrm{P}<0.01$ as compared to vehicle-treated group.

\section{DISCUSSION}

\section{CD31 expression}

CD31 is mainly expressed in the cytoplasm of endothelial cells and is normally used to evaluate angiogenesis in tumor research. Studies have shown that high CD31 expression is correlated with rapid tumor growth. Yang et al. (2009) found that ICA could increase tumor angiogenesis by increasing the expression of CD31 in the body. In our study, higher levels of CD31 were observed in all treatment groups compared to the vehicle-treated group. Previous studies suggested that VEGF expression in endometrial tissue is associated with microvessel density markers such as CD31 (Basilio-de-Oliveira and Pannain, 2015). Likewise, in this study, we found that CD31 and VEGF expression displayed similar expression patterns.

\section{Factor VIII expression}

Factor VIII, also called antihemophilic globulin, is a crucial blood clotting protein encoded by the F8 gene (Hoyer, 1981; Vehar et al., 1984). It is synthesized in the liver, and is composed of 2332 amino acid residues. Our results showed that the level of factor VIII was higher across all treatment groups compared to the vehicle-treated group, a trend similar to that observed with CD31 expression. Collectively, we speculate that ICA can increase the number of blood vessels in thin endometrium to enhance blood supply, resulting in an increase in endometrial thickness.

\section{VEGF expression}

Studies have found that VEGF can promote endometrial neovascularization by increasing the permeability of blood vessels, inducing vascular endothelial cell mitosis, 
and modulating gene expression in vascular endothelial cells (Ferrara, 2004; Hoeben et al., 2004; Wagatsuma et al., 2006; Miwa et al., 2009). Applanat et al. (2008) stated that estrogen can regulate the VEGF paracrine pathway, and can increase kinase insert receptor (VEGF receptor-2) expression in the endometrium. Koduri et al. (2006) suggested that expressions of VEGF and estrogen receptors are positively correlated. VEGF plays an important role in embryo implantation; insufficient VEGF has been observed in endometrial dysfunction and other unexplained causes of infertility. Additionally, significantly reduced expression of VEGF has been shown in animal models of thin endometrium (Binder et al., 2014; Hunter et al., 2015). Our study results showed that medium and high doses of ICA increase the expression of VEGF, CD31, and factor VIII. Furthermore, this effect was found to be dose-dependent. In groups treated with medium and high doses of ICA, significant increase in endometrial thickness, CK, and vimentin expression were observed as compared to those in the vehicle-treated group.

Interestingly, expression of VEGF, CD31, and factor III varied between the untreated control group and the $\mathrm{NaCl}$-injected vehicle group or the $95 \%$ ethanol-injected group. It is possible that $\mathrm{NaCl}$ lavage and $95 \%$ ethanol injection may have altered endometrial blood supply, which may have resulted in decrease of their expression. Therefore, we speculate that ICA promotes endometrial angiogenesis by increasing the expression of VEGF, CD31, and factor VIII.

PE exhibits dual effects as both an estrogen agonist and antagonist. The effect and dosage of PE, estrogen concentration in the body, and functional status of the target organ are all interdependent (Beck et al., 2003). We have previously shown that thin endometria exhibited low expression of ER and VEGF, even though the blood concentration of estrogen in the body remained normal (Le et al., 2015). In this study, VEGF, CD31, and factor VIII were significantly elevated in rats receiving medium and high doses of ICA, which was indicative of neovascularization. Moreover, CK and vimentin expression was increased in these groups, suggesting that repair of the endometrium was underway. Therefore, our results demonstrated that ICA can promote neovascularization and repair of endometrial tissue in rats with thin endometrium. Our previous research also revealed that PI3K and AKT were significantly reduced in thin endometria as compared to that in normal endometria, suggesting that the PI3K/AKT/eNOS signaling pathway may be involved in the pathology of thin endometrium (Le et al., 2016). However, the exact mechanism underlying this observation remains unclear.

In the present study, we found that ICA can promote thickening of thin endometria in rats. It is possible that ICA promotes endothelial cell proliferation, migration, and angiogenesis through the MEK/ERK and PI3K/Akt/eNOS pathways. Neovascularization of thin endometrium results in an increase in endometrial blood vessels and nutrition, thus promoting the development of the endometrium by breaking the Ichiro Miwa cycle, and thereby causing the endometrium to thicken.

\section{Conflicts of interest}

The authors declare no conflict of interest.

\section{ACKNOWLEDGMENTS}

Research supported by a grant from the Research Fund of Natural Science Foundation of Guangdong Province (\#2016A030313033).

Genetics and Molecular Research 16 (1): gmr16019126 


\section{REFERENCES}

Al-Ghamdi A, Coskun S, Al-Hassan S, Al-Rejjal R, et al. (2008). The correlation between endometrial thickness and outcome of in vitro fertilization and embryo transfer (IVF-ET) outcome. Reprod. Biol. Endocrinol. 6: 37. http:// dx.doi.org/10.1186/1477-7827-6-37

Applanat MP, Buteau-Lozano H, Herve MA and Corpet A (2008). Vascular endothelial growth factor is a target gene for estrogen receptor and contributes to breast cancer progression. Adv. Exp. Med. Biol. 617: 437-444. http://dx.doi. org $/ 10.1007 / 978-0-387-69080-3 \quad 42$

Aydin T, Kara M and Nurettin T (2013). Relationship between endometrial thickness and in vitro fertilizationintracytoplasmic sperm injection outcome. Int. J. Fertil. Steril. 7: 29-34.

Basilio-de-Oliveira RP and Pannain VLN (2015). Prognostic angiogenic markers (endoglin, VEGF, CD31) and tumor cell proliferation (Ki67) for gastrointestinal stromal tumors. World J. Gastroenterol. 21: 6924-6930.

Beck V, Unterrieder E, Krenn L, Kubelka W, et al. (2003). Comparison of hormonal activity (estrogen, androgen and progestin) of standardized plant extracts for large scale use in hormone replacement therapy. J. Steroid Biochem. Mol. Biol. 84: 259-268. http://dx.doi.org/10.1016/S0960-0760(03)00034-7

Binder NK, Evans J, Gardner DK, Salamonsen LA, et al. (2014). Endometrial signals improve embryo outcome: functional role of vascular endothelial growth factor isoforms on embryo development and implantation in mice. Hum. Reprod. 29: 2278-2286. http://dx.doi.org/10.1093/humrep/deu211

Chung BH, Kim JD, Kim CK, Kim JH, et al. (2008). Icariin stimulates angiogenesis by activating the MEK/ERK- and PI3K/Akt/eNOS-dependent signal pathways in human endothelial cells. Biochem. Biophys. Res. Commun. 376: 404408. http://dx.doi.org/10.1016/j.bbrc.2008.09.001

Ferrara N (2004). Vascular endothelial growth factor: basic science and clinical progress. Endocr. Rev. 25: 581-611. http:// dx.doi.org/10.1210/er.2003-0027

Gao Z (2011). Thin endometrium establishment and assessment in a rat model. Life Sci. Res. 15: 426-431.

Gleicher N, Kim A, Michaeli T, Lee HJ, et al. (2013). A pilot cohort study of granulocyte colony-stimulating factor in the treatment of unresponsive thin endometrium resistant to standard therapies. Hum. Reprod. 28: 172-177. http://dx.doi. org/10.1093/humrep/des370

Hoeben A, Landuyt B, Highley MS, Wildiers H, et al. (2004). Vascular endothelial growth factor and angiogenesis. Pharmacol. Rev. 56: 549-580.http://dx.doi.org/10.1124/pr.56.4.3

Hoyer LW (1981). The factor VIII complex: structure and function. Blood 58: 1-13.

Hunter RK, 2nd, Nevitt CD, Gaskins JT, Keller BB, et al. (2015). Adipose-derived stromal vascular fraction cell effects on a rodent model of thin endometrium. PLoS One 10: e0144823.http://dx.doi.org/10.1371/journal.pone.0144823

Kasius A, Smit JG, Torrance HL, Eijkemans MJ, et al. (2014). Endometrial thickness and pregnancy rates after IVF: a systematic review and meta-analysis. Hum. Reprod. Update 20: 530-541.http://dx.doi.org/10.1093/humupd/dmu011

Koduri S, Goldhar AS and Vonderhaar BK (2006). Activation of vascular endothelial growth factor (VEGF) by the ERalpha variant, ERDelta3. Breast Cancer Res. Treat. 95: 37-43. http://dx.doi.org/10.1007/s10549-005-9028-4

Lebovitz O and Orvieto R (2014). Treating patients with "thin" endometrium - an ongoing challenge. Gynecol. Endocrinol. 30: 409-414.http://dx.doi.org/10.3109/09513590.2014.906571

Le AW, Shan L, Wang ZH, Dai XY, et al. (2015). Effects of icariin on the expression of ER, VEGF, and KDR in the endometrial cells of thin endometrium. Genet. Mol. Res. 14: 11250-11258. http://dx.doi.org/10.4238/2015. September.22.19

Le AW, Shan LL, Dai XY, Xiao TH, et al. (2016). PI3K, AKT, and P-AKT levels in thin endometrium. Genet. Mol. Res. 15: 1-10 http://dx.doi.org/10.4238/gmr.15017184.

Luo Z, Liu M, Sun L and Rui F (2015). Icariin recovers the osteogenic differentiation and bone formation of bone marrow stromal cells from a rat model of estrogen deficiency-induced osteoporosis. Mol. Med. Rep. 12: 382-388.

Miwa I, Tamura H, Takasaki A, Yamagata Y, et al. (2009). Pathophysiologic features of "thin" endometrium. Fertil. Steril. 91: 998-1004.http://dx.doi.org/10.1016/j.fertnstert.2008.01.029

Shufaro Y, Simon A, Laufer N and Fatum M (2008). Thin unresponsive endometrium - a possible complication of surgical curettage compromising ART outcome. J. Assist. Reprod. Genet. 25: 421-425. http://dx.doi.org/10.1007/s10815-008$\underline{9245-\mathrm{y}}$

Vehar GA, Keyt B, Eaton D, Rodriguez H, et al. (1984). Structure of human factor VIII. Nature 312: 337-342. http:// dx.doi.org/10.1038/312337a0

Wagatsuma A, Tamaki H and Ogita F (2006). Sequential expression of vascular endothelial growth factor, Flt-1, and KDR/ Flk-1 in regenerating mouse skeletal muscle. Physiol. Res. 55: 633-640.

Xue L, Wang Y, Jiang Y, Han T, et al. (2012). Comparative effects of er-xian decoction, epimedium herbs, and icariin with estrogen on bone and reproductive tissue in ovariectomized rats. Evid. Based Complement. Alternat. Med. 2012: 241416. http://dx.doi.org/10.1155/2012/241416

Yang JX, Fichtner I, Becker M, Lemm M, et al. (2009). Anti-proliferative efficacy of icariin on HepG2 hepatoma and its possible mechanism of action. Am. J. Chin. Med. 37: 1153-1165. http://dx.doi.org/10.1142/S0192415X09007569

Genetics and Molecular Research 16 (1): gmr16019126 